THE ASTROPHYSICAL JOURNAL, 474:L143-L145, 1997 January 10

- 1997. The American Astronomical Society. All rights reserved. Printed in U.S. A.

NASA-CR-204720

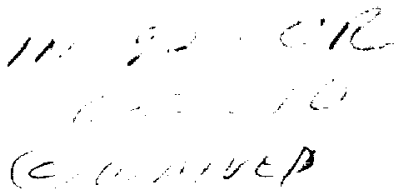

\title{
SELF-CONSISTENT AND TIME-DEPENDENT SOLAR WIND MODELS
}

\author{
K. K. Ong, ${ }^{1}$ Z. E. Musielak, ${ }^{1}$ R. Rosner, ${ }^{2}$ S. T. Suess, ${ }^{3}$ and M. E. Sulkanen ${ }^{3}$ \\ Received 1996 July 15; accepsed 1996 October 25
}

\begin{abstract}
We describe the first results from a self-consistent study of Alfvén waves for the time-dependent, single-fluid magnetohydrodynamic (MHD) solar wind equations, using a modified version of the ZEUS MHD code. The wind models we examine are radially symmetrical and magnetized; the initial outflow is described by the standard Parker wind solution. Our study focuses on the effects of Alfvén waves on the outflow and is based on solving the full set of the ideal nonlinear MHD equations. In contrast to previous studies, no assumptions regarding wave linearity, wave damping, and wave-flow interaction are made; thus, the models naturally account for the back-reaction of the wind on the waves, as well as for the nonlinear interaction between different types of MHD waves. Our results clearly demonstrate when momentum deposition by Alfvén waves in the solar wind can be sufficient to explain the origin of fast streams in solar coronal holes; we discuss the range of wave amplitudes required to obtained such fast stream solutions.
\end{abstract}

Subject headings: MHD - solar wind - waves

\section{INTRODUCTION}

The fact that the solar wind can be divided into fast and slow streams has been known for many years. Fast streams are associated with solar coronal holes, velocities in the range $600-800 \mathrm{~km} \mathrm{~s}^{-1}$, and relatively uniform physical properties. Slow streams are associated with coronal sector boundaries, velocities in the range $300-500 \mathrm{~km} \mathrm{~s}^{-1}$, and highly variable physical properties. In this Letter, we restrict our attention to fast streams because the time-varying properties of slow streams suggest that the latter are not in equilibrium with the coronal base, an issue that is beyond the scope of this Letter.

The main difficulty with understanding fast solar wind streams is that their origin cannot be explained (e.g., Axford \& McKenzie 1992) simply on the basis of the theory of thermally driven solar winds proposed by Parker $(1958,1965)$. An additional momentum deposition mechanism is required to account for the onset of such high-speed flows. It has been suggested that the additional force is supplied by Alfvén waves (Hollweg 1978). A number of calculations have already been made to investigate the behavior of Alfvén waves in the solar wind and to construct solar wind models based on Alfvén wave momentum deposition (Alazraki \& Couturier 1971; Hollweg 1973; 1978; Jacques 1978; Heinemann \& Olbert 1980; Leer, Holzer, \& Flå 1982; MacGregor \& Charbonneau 1994; Lau \& Siregar 1996). Similar ideas have also been used to explain the acceleration of cool massive winds observed from late-type giants and to construct models of these winds (Belcher \& Olbert 1975; Hartmann \& MacGregor 1980, 1982; Holzer, Flå, \& Leer 1983; Hartmann \& Avrett 1984; An et al. 1989, 1990; Barkhudarov 1991; Rosner et al. 1991, 1995; Lou 1993; Velli 1993). The basic challenge for these solar and stellar models is that one must account for the onset of the mass loss, the mass-loss rate, and the terminal speed, and at the same time, explain the observed heating in the region of the atmosphere

\footnotetext{
${ }^{1}$ Center for Space Plasma, Aeronomic, and Astrophysics Research, University of Alabama in Huntsville, Huntsville, AL 35899.

2 Deparment of Astronomy and Astrophysics, University of Chicago, Chicago, IL 60637.

${ }^{3}$ Space Science Laboratory, NASA Marshall Space Flight Center, Huntsville, AL 35812.
}

where the wind originates. The obtained results demonst al that, in general, it is possible to construct models based o wave heating and momentum deposition by Alfvén waves, bu as pointed out by Holzer et al. (1983), such models suffer fror the defect that the necessary wave-damping lengths are nc only ad hoc, but also need to be extremely finely tuned $i$ value. A possible resolution to this difficulty has been prc posed by An et al. (1990) and Rosner et al. (1991), wh suggested that reflection and trapping of non-WKB Alfvé waves in the solar and stellar atmospheres may lead to significant increase in the acceleration force, and that thi force may be sufficient to explain the acceleration of wind (see Rosner et al. 1995). However, recent numerical (Krogule et al. 1994; MacGregor \& Charbonneau 1994) and analytice (Lou \& Rosner 1994) results indicate that the push on th. background medium exerted by non-WKB Alfvén waves i lower than the corresponding force exerted by WKB waves Similar results have been also reported by Ong, Krogulec, $d$ Musielak (1995), who constructed the first purely theoretical self-consistent, time-dependent wind models based on mo mentum deposition by linear Alfvén waves.

The preliminary results reported by Ong et al. (1995 demonstrate that momentum deposition by linear Alfié waves can lead to a slow secular increase in wind velocity However, although the cumulative wave effects can be see! clearly, they are not prominent, and the maximum increase it the wind velocity in such models only reaches $\approx 10 \%$ of it initial value before the solution approaches a steady state Therefore, we can conclude that small-amplitude (linear Alfvén waves cannot contribute enough momentum to thi solar wind to explain the observed properties of fast streams This indicates that models must be extended to treat finite amplitude, nonlinear Alfvén waves. Unfortunately, such at extension cannot be done in a one-dimensional approach, bu instead requires (at least) two-dimensional numerical calcula tions. In this Letter, we report on our first theoretical self-consistent, time-dependent wind models based on mo mentum deposition by nonlinear Alfvén waves. The detailec procedure for constructing these models is described in $\S 2$. 


\section{WIND MODELS}

To calculate our wind models, we start with a standard Parker model of thermally driven winds (Parker 1958) and treat it as the initial flow. The structure of an atmosphere computed with this initial flow is then perturbed by radially propagating toroidal Alfvén waves of a finite amplitude. The interaction between these waves and the flow is treated self-consistently; e.g., the structure of the wind and atmosphere is modified by the waves and, in turn, the wave behavior is influenced by this newly modified structure. The physical parameters describing the background medium are recorded (as they fluctuate on timescales of the Alfvén wave period) and then time-averaged over the Alfvén wave crossing time in order to obtain estimates for the corresponding parameter values of the flow on timescales long when compared to the Alfvén wave period.

In order to perform these calculations, we modified the original ZEUS code (Stone \& Norman 1992a, 1992b; see also Ong 1997), and we use it to solve the full set of ideal single-fluid compressible MHD equations in two-dimensions; we consider a single magnetic flux tube along which the field decreases radially. At the lower boundary of our computational domain the field line is perturbed in such a way that toroidal $(\partial / \partial \phi=0)$ Alfvén waves are continuously generated. The upper boundary is transparent, which means that the wind and waves freely leave the computational domain. Since we are solving the single-fluid MHD equations without any further simplifications, processes such as wave reflection, nonlinear coupling between the various MHD waves, and shock formation are automatically accounted for. For any given solar wind model, it is necessary to specify the strength of the ambient (background) magnetic field, $B_{0}$, the wind temperature, $T_{0}$, and wind density, $\rho_{0}$, at the base of the coronal ( $r=1 R_{\odot}$ ), and to prescribe the initial flow. The results presented in this paper have been obtained using $B_{0}=10 \mathrm{G}$, $T_{0}=1.0 \times 10^{6} \mathrm{~K}$, and $\rho_{0}=1.3 \times 10^{-15}$. The initial flow's velocity is $V=0.2 \mathrm{~km} \mathrm{~s}^{-1}$ at $r=1 R_{\odot}$, becomes supersonic at $r=37.8 R_{\odot}$, and reaches a velocity of $V=418 \mathrm{~km} \mathrm{~s}^{-1}$ at $r=91 R_{\odot}$. A source of Alfvén waves is located at the base of the corona; we assume that this source generates continuous, monochromatic, sinusoidal waves with period of $1 \mathrm{hr}$. We considered four different values of the wave amplitude, corresponding to three different wave amplitude regimes: the essentially linear case $\left(8 \mathrm{~km} \mathrm{~s}^{-1}\right)$; the weakly nonlinear, finite amplitude case $\left(20 \mathrm{~km} \mathrm{~s}^{-1}, 40 \mathrm{~km} \mathrm{~s}^{-1}\right)$; and the fully nonlinear case $\left(75 \mathrm{~km} \mathrm{~s}^{-1}\right)$. In all of these cases, the computational domain extended from $r=1 R_{\odot}$ (the base of the corona) to $r=91 R_{\odot}$; the calculations were continued for $278 \mathrm{hr}$, sufficient time to allow the waves to interact with the initial flow and to change its physical properties. We note again that the results we show are time averages of the wind velocity over timescales long when compared to the Alfvén wave period.

\section{RESULTS AND DISCUSSION}

The results of our numerical simulations are presented in Figure 1, which shows the distribution of the time-averaged wind velocity with distance. It is clearly seen that linear Alfven waves, with amplitudes comparable to, or smaller than, $10 \mathrm{~km} \mathrm{~s}^{-1}$, add very little momentum to the initial flow; the resulting wind velocity at $r=91 R_{\odot}$ is $423 \mathrm{~km} \mathrm{~s}^{-1}$, which is only $4 \mathrm{~km} \mathrm{~s}^{-1}$ higher than the thermally driven initial flow. It must be noted that a higher wind velocity is obtained when a time-averaged WKB wind type of model is used (e.g., MacGre-

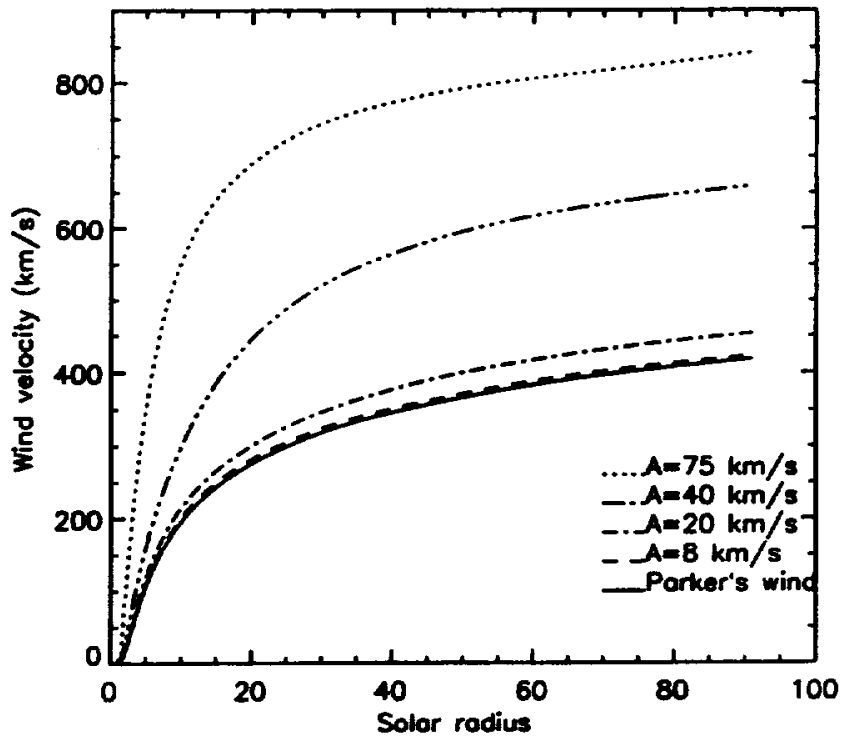

FIG. 1.-Time-averaged wind velocity profiles for four different solar wind models. In each model, the initial flow is assumed to be the thermally driven (Parker) wind. Alfuen waves with the same period $(1 \mathrm{hr})$ but different amplitudes, $A$, are generated at the base of each model (corresponding to the base of solar coronal boles) and allowed to interact with the initial flow for 278 hr. After this time, the resulting time-averaged wind velocity profiles are calculated. The obtained results show that the presence of Alfven waves with amplitudes ranging from 40 to $75 \mathrm{~km} \mathrm{~s}^{-1}$ is required in the solar wind in order to explain the origin of fast streams.

gor \& Charbonneau 1994). The difference can be explained by the presence of non-WKB effects for Alfvén waves with period of $1 \mathrm{hr}$ considered in our calculations. Much more momentum is deposited by finite amplitude Alfvén waves; waves with amplitude $20 \mathrm{~km} \mathrm{~s}^{-1}$ increase the wind velocity at $r=91 R_{\odot}$ to $453 \mathrm{~km} \mathrm{~s}^{-1}$ ( $34 \mathrm{~km} \mathrm{~s}^{-1}$ above Parker's wind solution), while waves with amplitude $40 \mathrm{~km} \mathrm{~s}^{-1}$ increase the wind velocity at $r=91 R_{\odot}$ to $660 \mathrm{~km} \mathrm{~s}^{-1}$, which is $241 \mathrm{~km} \mathrm{~s}^{-1}$ above the velocity of the thermally driven background flow. Finally, the results shown in Figure 1 demonstrate that nonlinear Alfvén waves can deposit enough momentum to increase the velocity of the initial outflow at $r=91 R_{\odot}$ to $845 \mathrm{~km} \mathrm{~s}^{-1}$, which is $427 \mathrm{~km} \mathrm{~s}^{-1}$ above Parker's wind solution. This is an interesting result, since it shows that Alfvén waves with amplitudes ranging from 50 to $80 \mathrm{~km} \mathrm{~s}^{-1}$ can, in principle, account for the observed velocities of fast streams in the solar wind.

The results presented in this Letter lead to two important conclusions. By constructing theoretical self-consistent, timedependent solar wind MHD models based on momentum deposition by Alfvén waves, we have shown that, first, models based on momentum deposition by small-amplitude (linear) Alfvén waves cannot account for the basic observational properties of fast streams in the solar wind; and second, that in order to explain the observed range of the wind velocities of fast streams $\left(600-800 \mathrm{~km} \mathrm{~s}^{-1}\right)$, one has to assume Alfvén wave amplitudes ranging from 40 to $75 \mathrm{~km} \mathrm{~s}^{-1}$ at the coronal base of the solar wind. This range of required Alfven wave amplitudes seems to be in agreement with observations of line broadening of $\mathrm{Mg}$ coronal emission lines (Hassler et al. 1990) and with more recent Ulysses observations (e.g., Phillips et al. 1995).

We thank M. Cuntz, Y. Q. Lou, and R. L. Moore for many discussions. This work was supported in part by the NASA Astrophysics Theory Program (grant NAG5-3027) at the 
University of Alabama in Huntsville (K. K. O., Z. E. M., and R. R.), the NASA Space Plasma Theory Program at the University of Chicago (R. R., Z. E. M., and K K. O.), NSF under grant ATM-9526196 (K. K. O. and Z. E. M.), by th X-Ray Astronomy Branch at NASA/MSFC (K.K. O.), and I the Ulysses Project (S. T. S.).

\section{REFERENCES}

Alazraki, G., \& P. Cauterier, P. 1971, A\&A, 13, 380

An, C. H., Musielak, Z. E., Moore, R. L, \& Suess, S. T. 1989, ApJ, 345, 597

An, C. H., Suess, S. T., Moore, R. L., \& Musielak, Z. E. 1990, ApJ, 350, 309

Axford, W. I., \& McKenzie, J. F. 1992, Solar Wind Seven, ed. E. Marsch \& R. Schwenn (Oxford: Pergamon Press), 1

Barkhudarov, M. R. 1991, Sol. Phys., 135, 131

Belcher, J., \& Olbert, S. 1975 ApJ, 200, 369

Hartmann, L., \& Avrett, E. H. 1984, ApJ, 284, 238

Hartmann, L., \& MacGregor, K. B. 1980, ApJ, 242, 260

. 1982, ApJ, 257, 264

Hassler, D. M., Rottman, G. J., Shoub, E. C., \& Holzer, T. E. 1990, ApJ, 348, L77

Heinemanm, M., \& Olbert, S. 1980, J. Geophys. Res., 95, 1311

Hollweg, J. V. 1973, ApJ, 181, 547

1978, Rev. Geophys. Space Phys., 16, 689

Holzer, T. E., Flå. T., \& Leer, E. 1983, ApJ, 275, 808

Jacques, S. A. 1978, ApJ, 226, 632

Krogulec, M., Musielak, Z. E., Suess, S. T., Nerney, S. F., \& Moore, R. L. 1994 J. Geophys. Res., 99, 23489
Lau, Y.-T., \& Siregar, E. 1996, ApJ, 465, 451

Leer, E., Holzer, T. E., \& Fla, T. 1982, Space Sci. Rev., 33, 161

Lou, Y. Q. 1993, J. Geophys. Res., 98, 3563

Lou, Y. Q., \& Rosner, R. 1994, ApJ, 424, 429

MacGregor, K B., \& Charbonneau, P. 1994, ApJ, 430, 387

Ong, K. K 1997, ApJ, submitted

Ong. K. K. Krogulec, M., \& Musielak, Z. E. 1995, Bull. AAS, 27(2), 975

Parker, E. N. 1958, ApJ, 128, 664

1965, Space Sci. Rev., 4, 666

Phillips, J. L., et al. 1995, Science, 268, 1030

Rosner, R., An, C. H., Musielak, Z. E., Moore, R. L., \& Suess, S. T. 1991, If 372, L91

Rosner, R., Musielak, Z. E., Cattaneo, F., Moore, R. L., \& Suess, S. T. 195 ApJ, $442, \mathrm{~L} 25$

Stone, J. M., \& Norman, M. L. 1992a, ApJS, 80, 753

-1992b, ApJS, 80,791

Velli, M. 1993, A\&A, 270, 304 
